\title{
Predict-First Experiments and Modeling of Perturbative Cold Pulses in the DIII-D Tokamak
}

\author{
P. Rodriguez-Fernandez, ${ }^{1 *}$ A. E. White, ${ }^{1}$ N. T. Howard, ${ }^{1}$ B. A. Grierson, ${ }^{2}$ L. Zeng, ${ }^{3}$ X. Yuan, ${ }^{2}$ \\ G. M. Staebler, ${ }^{4}$ M. E. Austin, ${ }^{5}$ T. Odstrcil, ${ }^{1}$ T. L. Rhodes,${ }^{3}$ F. Sciortino, ${ }^{1}$ J. E. Rice, ${ }^{1}$ K. E. Thome, ${ }^{4}$ \\ C. Angioni, ${ }^{6}$ E. Fable, ${ }^{6}$ O. Meneghini ${ }^{4}$
}

${ }^{1}$ MIT Plasma Science and Fusion Center, Cambridge, Massachusetts 02139, USA

${ }^{2}$ Princeton Plasma Physics Laboratory, Princeton, New Jersey 08540, USA

${ }^{3}$ Department of Physics and Astronomy, University of California-Los Angeles, Los Angeles, California 90095, USA

${ }^{4}$ General Atomics, P. O. Box 85608, San Diego, California 92186-5608, USA

${ }^{5}$ Institute for Fusion Studies, University of Texas-Austin, Austin, TX 78712, USA

${ }^{6}$ Max Planck Institut für Plasmaphysik, D-85748 Garching, Germany

*Email: pablorf@mit.edu

\begin{abstract}
Cold pulses are introduced in Ohmic DIII-D tokamak plasmas, revealing for the first time in this machine a quick increase of core electron temperature shortly after the edge cold-pulse injection at low collisionality. The experimental results are consistent with predict-first simulations enabled by the Trapped Gyro-LandauFluid (TGLF) transport model. Measurements of electron density evolution during the cold-pulse propagation are enabled by a high time resolution density profile reflectometer. The density evolution reveals the quick propagation of a pulse from edge to core, which is a mechanism to transiently increase core temperature in low-collisionality plasmas. Local transport simulations with measured density evolution demonstrate that the core temperature response can indeed be explained by the stabilization of Trapped Electron Mode (TEM) turbulence at low collisionality, thus providing confidence that local transport modeling is enough to explain cold-pulse propagation and associated phenomenology.
\end{abstract}

\section{Introduction}

Cold-pulse experiments and associated phenomena have been puzzling plasma transport physicists for more than twenty years. In 1995, an experimental study in the TEXT tokamak [1] showed evidence of rapid core temperature increases following the injection of perturbative cold pulses at the edge of lowcollisionality plasmas. Such unexplained behavior was reproduced in many other tokamaks and helical 
devices (TFTR [2], Tore Supra [3], RTP [4], ASDEX Upgrade [5], JET [6], LHD [7], HL-2A [8], Alcator C-Mod [9], KSTAR [10] and J-TEXT [11]).

The importance of whether or not transport models can explain and reproduce cold-pulse propagation is clear: why should a transport model be trusted for prediction of future burning plasmas if it fails to explain such a robust and reproducible experiment? The speed and the change of sign of the perturbation led transport physicists to consider these phenomena as evidence of nonlocal transport. In the framework of turbulence, nonlocal effects refer to mechanisms that allow transport fluxes at a given position in the plasma to be driven by pressure gradients and other parameters at a distance longer than a few radial correlation lengths of the turbulence $[12,13]$. As such, nonlocal effects could, in principle, allow for core transport responses that are independent from mean local plasma parameters at the same position in space and at the same time [14]. The violation of the local closure could potentially give rise to transport hysteresis, and was justified by the fact that the core temperature increase happens faster than the change on any other plasma quantity. Furthermore, the "reversed polarity" of the core response (temperature increase rather than a drop) would violate the assumption of diffusive heat transport driven by local temperature gradients.

However, while nonlocal effects could exist in certain regimes, such a strong and robust response of the plasma core being driven by nonlocal phenomena would mean that the fundamental assumptions of turbulent transport models would need to be revisited [14, 15]. Such models, like TGLF [16] and QuaLiKiz [17], among others, are widely used to predict plasma performance and profiles in ITER and future reactors $[18,19,20,21,22,23]$, and have been extensively validated against experiments $[18,24$, 25, 26, 27]. Turbulent heat transport, as provided by these models, may be driven by plasma parameters other than temperature gradients, exhibit critical-gradient behavior and may be strongly nonlinear (e.g. stiff transport [28]). An example of this is the case of Trapped Electron Mode (TEM) turbulence, which is well known to drive large amounts of electron heat flux and can lead to hysteresis with respect to the electron temperature gradient. The possibility of such multi-channel interactions and high transport stiffness opens new pathways for understanding perturbative transport phenomena.

For this reason, and twenty years later, cold pulses have been revisited from the standpoint of both modeling and experiment. A recent study [29] provided a feasible explanation to the cold-pulse phenomena from a local transport perspective. Under the assumptions that (1) the plasma core at low collisionality is dominated by TEM-type turbulence, and (2) a density perturbation exists and propagates inwards after the injection of particles at the edge, then the core electron temperature could increase as a 
result of turbulence stabilization. While the former assumption fits within many experimental observations and simulations of low collisionality plasmas $[30,31,25]$, the latter remains to be validated. Because of the rapid cold-pulse behavior, experiments in Alcator C-Mod could only provide line-integrated measurements of electron density, and that was used as the only constraint to the modeled density perturbation.

The model has been successful in explaining many experimental trends in Alcator C-Mod plasmas [32], but the phenomenology of cold-pulse propagation is very rich and other experimental observations still remain to be addressed. Among them, the transition condition for cold-pulse behavior seems to be affected by a $1 / R$ dependence $[9,33]$, with $R$ as the major radius. Using unique pre-experiment predictions with the Trapped Gyro-Landau-Fluid (TGLF) model, and new LBO-enabled experiments and analysis, this paper aims at answering the open question of whether simulations in a device other than Alcator CMod can reproduce the transition condition (thus able to capture the $1 / R$ dependence of the empirical scaling), as well as to address the open question of the propagation of density perturbations in experiments.

The organization of this paper is as follows. In section 2, this paper summarizes current understanding of cold-pulse phenomenology within the framework of local drift-wave turbulent transport. In Section 3 , predictions for cold-pulse dynamics in the DIII-D tokamak [34] are described. These theory-based predictions were used to design new cold-pulse experiments at DIII-D. Section 4 describes the experiments in the DIII-D tokamak to track cold-pulse propagation enabled by the recently installed laserblow-off (LBO) system, and the high-time resolution ECE radiometer and profile reflectometer. Section 5 describes the post-experiment modeling, and specifically addresses the question of whether a density pulse exists within experimental error bars that can provide the observed core temperature behavior. Section 6 summarizes the results and discusses future directions.

\section{Cold-Pulse Phenomenology and Open Questions}

Modeling of Alcator C-Mod cold-pulse experiments found that the reduction of core electron and impurity density gradients and main ion dilution, and the increase of effective ion charge and collisionality were the mechanisms by which TEMs are stabilized at the plasma core, leading to temperature increase [32]. These effects can be viewed as the consequence of the injection of impurities, subsequent propagation to the plasma core, and quasineutrality. In Alcator C-Mod, cold-pulse simulations were performed by constraining the density pulse with the following requirements: (1) line-integrated density 
is within error bars of the two-color interferometer measurements during the cold-pulse propagation, (2) the pulse originates at the edge and propagates inwards. Results in Alcator C-Mod [29] demonstrated that such a density pulse can lead to the observed temperature behavior. This model could also explain the disappearance of the temperature inversion effect at high density [33 and references therein], the trend with plasma current [35], the spatial correlation between temperature flex point and rational surfaces [36, 9], and the connection to thermal coupling between ions and electrons [12]. Recent work at ASDEX Upgrade [37] shows that the effect of auxiliary heating on cold-pulse behavior [5, 38, 39, 40, 6] can also be explained by local models like TGLF, as well as the fast reduction of normalized core density gradient following the cold-pulse injection.

At high density, three main effects are known to contribute to the transition to dominant Ion Temperature Gradient (ITG) driven turbulence: higher collisionality, reduction in impurity content and increase in normalized ion temperature gradient. The dominance of ITG turbulence suppresses the stabilizing effect of core density gradients following the cold-pulse injection. Consequently, the electron temperature drop can propagate to the core without any "inversion". In simulations, it is also observed that core ion temperature gradient may also transiently increase [29], an effect of higher edge collisional coupling between ions and electrons at high density.

At high plasma current, collisionality decreases (at constant density), leading to less de-trapping and more TEM activity, which covers larger portions of the plasma. Other effects, such as the reduction of normalized ion temperature gradient $a / L_{T_{i}}$ (less collisional exchange, only source of heat for the ions in Ohmic plasmas, generally leads to flatter $T_{i}$ ) may also result in prevalence of TEM over ITG turbulence. This dependence of dominant microinstability on plasma current is also compatible with the upshift of the critical density for ohmic confinement saturation [41, 9, 42], and explains the existence of temperature inversions at high density when the plasma current is increased [35]. Such a model could also explain why the temperature flex point moves outwards (along with rational surfaces) when the current increases [36, 9].

As stated in the introduction, this paper studies whether this model can also capture the behavior of cold-pulse propagation in the DIII-D tokamak and whether the fast density propagation used in the model is consistent with local experimental measurements. 


\section{Predictions of Cold-Pulse Behavior in Ohmic DIII-D Plasmas}

A database of cold-pulse experiments in different machines $[9,33]$ suggests that the transition in core transport behavior (from "temperature inversion effect" to "standard temperature drop") happens at constant collisionality (as given by $n_{e} q_{95} R=$ const). Machines with similar size as DIII-D experience temperature inversions below $n_{e} q_{95} \approx 12.0 \cdot 10^{19} \mathrm{~m}^{-3}$ for ASDEX Upgrade [5] and below $n_{e} q_{95} \approx 6.0$. $10^{19} \mathrm{~m}^{-3}$ for HL-2A [43]. Such large uncertainty in the transition condition in DIII-D motivated the use of predict-first theory-based simulations of the core transport behavior.

An extensive search over recent Ohmic low-density DIII-D plasmas leads to the identification of a discharge with $n_{e} q_{95}$ below both ASDEX Upgrade and HL-2A thresholds. Specifically, a DIII-D plasma with $n_{e} q_{95} \approx 4.4 \cdot 10^{19} \mathrm{~m}^{-3}$ is selected as the baseline. Next, perturbations in density and radiation are introduced in the simulation to mimic a laser blow-off injection. Lacking experimental data of the perturbations in DIII-D, we scaled down the pulses in density and radiation profiles from Alcator C-Mod. In particular, given that this DIII-D plasma had 8-fold lower absolute density than the lowcollisionality plasma in Alcator C-Mod [29], the perturbations were reduced

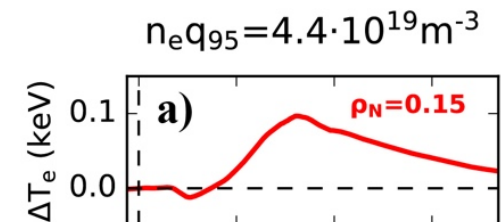

$$
\mathrm{n}_{\mathrm{e}} \mathrm{q}_{95}=13.2 \cdot 10^{19} \mathrm{~m}^{-3}
$$
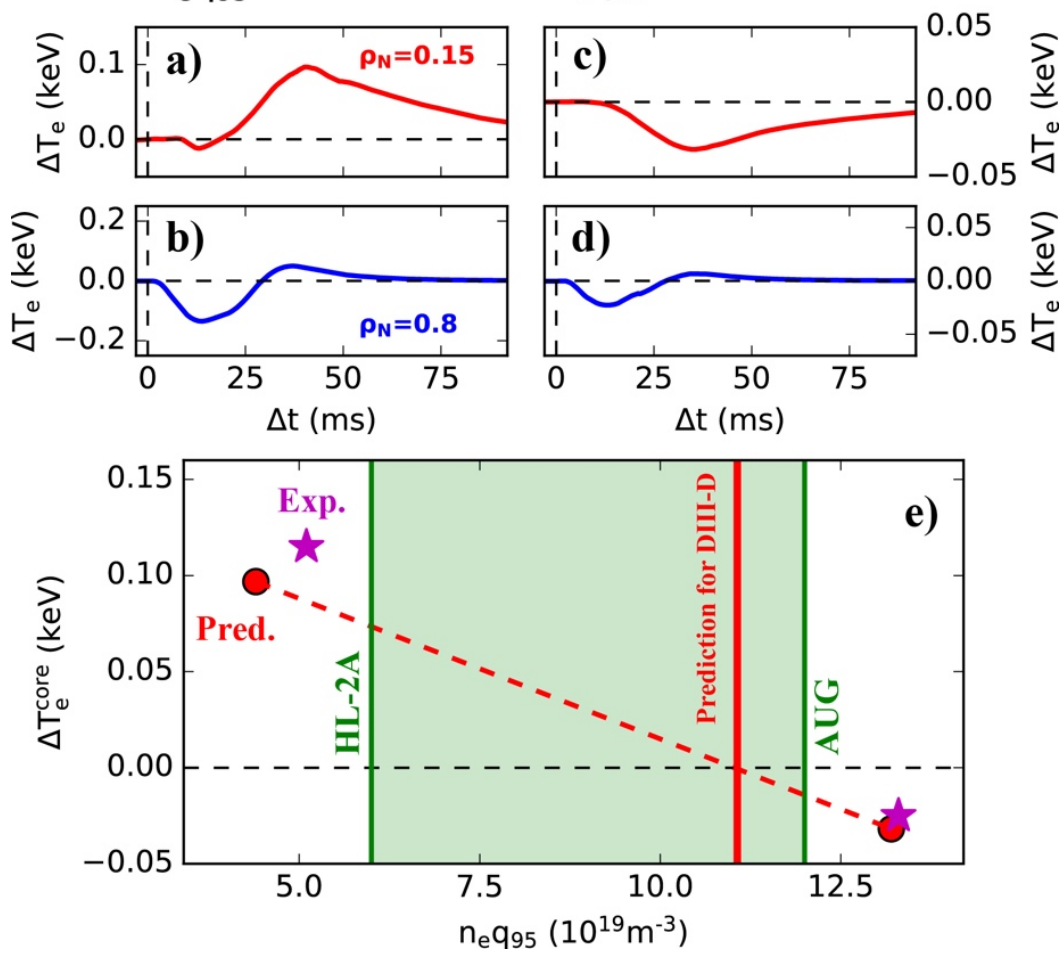

Figure 1. Prediction of changes in edge and core electron temperature at low $(a, b)$ and high $(c, d)$ collisionality in DIII-D. (e) Comparison between empirical and theory-based prediction of the transition condition for DIII-D. Green area indicates the uncertainty in the empirical scaling of the transition for DIII-D. Experiment (run after the predictions were made) is also plotted.

accordingly ( 8 times smaller density perturbation and $8^{2}$ lower radiation). A perturbative transport simulation identical to that in Ref. [29] was performed with the baseline discharge $\left(n_{e} q_{95} \approx 4.4\right.$. $\left.10^{19} \mathrm{~m}^{-3}\right)$ and a higher collisionality version of the baseline $\left(n_{e} q_{95} \approx 13.2 \cdot 10^{19} \mathrm{~m}^{-3}\right)$, constructed by simply scaling up the density profile. 
Fig. 1 shows that these simulations confirm the existence of the two different core transport behaviors in DIII-D: inversion versus non-inversion of the core electron temperature. Following the edge temperature drop (Fig. 1b and Fig. 1d), the core temperature increases at low collisionality (Fig. 1a) and decreases at higher collisionality (Fig. 1c). Under the assumption that the magnitude of the temperature inversion decreases linearly with density $[35,32]$, a theory-based predict-first transition is obtained by linearly interpolating the magnitude of the core temperature change for the two simulations. A transition condition of $n_{e} q_{95} \approx 11.1 \cdot 10^{19} \mathrm{~m}^{-3}$ is found for DIII-D. Fig. 1e illustrates the transition conditions for ASDEX Upgrade and HL-2A (in green) and the predict-first transition for DIII-D (in red).

\section{Experiments in Ohmic DIII-D Plasmas}

Based on the predictions, two experimental conditions were designed that would exhibit both core transport behaviors in the DIII-D tokamak. The two plasmas presented here had lower-single-null diverted geometry, plasma current $I_{p}=1.0 \mathrm{MA}$, magnetic field $B_{T}=2.0 \mathrm{~T}$, and safety factor $q_{95}=5.1$. Both plasmas were Ohmic, with laser blow-off injections at $t=1800 \mathrm{~ms}$, and diagnostic neutral beams at $t=$ $1980 \mathrm{~ms}$. As shown in Fig. 2a, at the moment of the LBO injection, the first plasma had a line-averaged density $\overline{n_{e}} \simeq 1.0 \cdot 10^{19} \mathrm{~m}^{-3}$ (from now on, "low density" plasma) and the second one had $\overline{n_{e}} \simeq 2.6$. $10^{19} \mathrm{~m}^{-3}$ ("high density"). This choice of parameters provided plasmas with $n_{e} q_{95} \simeq 5.1 \cdot 10^{19} \mathrm{~m}^{-3}$ at low density and $n_{e} q_{95} \simeq 13.3 \cdot 10^{19} \mathrm{~m}^{-3}$ at high density, thus covering the empirical and the theory-based predictions of the transition condition. Neutral beams were not used during the cold-pulse propagation so that the temperature inversion does not vanish, which is observed in experimental $[40,6]$ and modeling [37] studies.

Electron temperature and density traces were measured with a fast time-resolution ECE radiometer [44] and a density profile reflectometer [45], respectively. Ion temperature, impurity density and toroidal rotation profiles were measured with a CER system [46, 47]. To trigger cold pulses, the LBO system introduces non-intrinsic non-recycling impurities $\left(\sim 10^{18}\right.$ particles $)$ that reduce the edge electron temperature by locally enhancing radiative losses at the plasma edge. In this experiment, aluminum was chosen as the LBO impurity, and the amount to be introduced was approximately the same for both plasmas. 


\section{i. Core Temperature Behavior}

Fig. $2 \mathrm{~b}$ demonstrates that the discharges exhibit two different core transport behaviors: following the edge cold-pulse injections (Fig. 2c), the core temperature abruptly increases in the experiment at low density and decreases at high density. At high density, the edge perturbation is smaller $\left(\Delta T_{e} \approx\right.$ $-30 \mathrm{eV}$ versus $\Delta T_{e} \approx-75 \mathrm{eV}$ at low-density), which could be related to the higher collisional coupling between plasma species, and the lower radiative losses relative to the plasma stored energy, as the same amount of aluminum impurities was introduced in both plasmas. However, past work in Alcator C-Mod [35] suggested that the amount of impurities should not significantly change the qualitative features (i.e. inversion versus noninversion) of the core electron temperature behavior. At low density, the core temperature increases $\Delta T_{e} \approx 115 \mathrm{eV}$, while at high density it drops by $\Delta T_{e} \approx-25 \mathrm{eV}$. The peak of the temperature rise at low density is reached $\Delta t \approx$ $50 \mathrm{~ms}$ after the injection, which is approximately an energy confinement time (estimated to be in this plasma $\tau_{E} \approx 60 \mathrm{~ms}$ ). The lowest point of the core temperature drop at high density is reached $\Delta t \approx 50 \mathrm{~ms}$ after injection, which instead is significantly shorter than an energy confinement time (estimated as $\tau_{E} \approx 120 \mathrm{~ms}$ ). Notably, the magnitudes of the temperature increase at low density and temperature drop at high density follow closely the behavior predicted by the model before the experiment was performed, as depicted in Fig. 1e (magenta stars).

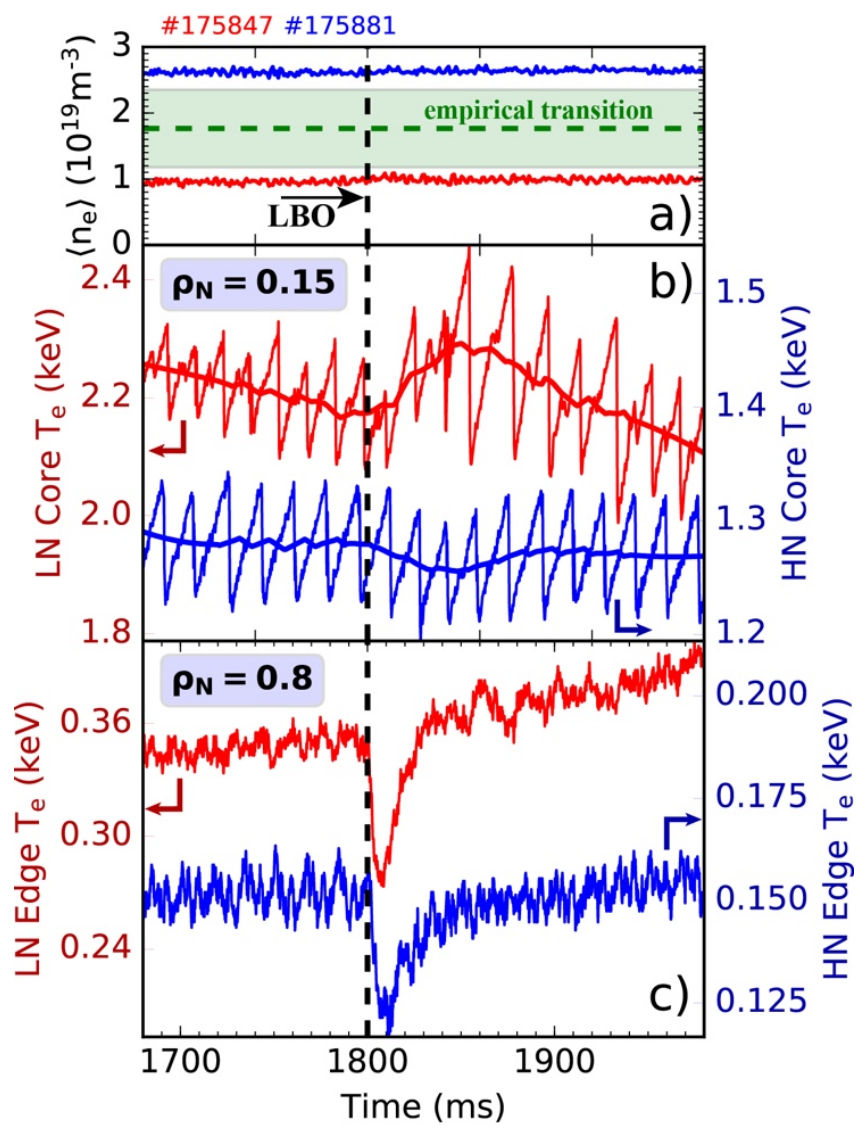

Figure 2. a) Line-average density, b) Core temperature $\left(\rho_{N}=\right.$ $0.15)$, and c) Edge temperature $\left(\rho_{N}=0.8\right)$ for low (red, $\left.L N\right)$ and high density (blue, HN) shots. Core temperatures have also been filtered for visualization purposes. Green dashed line in a) indicates the expected transition density as given in [33] for $q_{95}=5.1$. Shaded green area covers range of transition density for similar size machines, $A U G$ and $H L-2 A$. 


\section{ii. Evidence of Fast Density Pulse}

For the first time, direct measurements of the density evolution following the edge cold pulse were made by a high time resolution density profile reflectometer system, as depicted in Fig. 3 for the low density discharge discussed in Section 4i. A perturbation in electron density is observed to travel from edge (where impurities are deposited) to core in a remarkably fast time-scale. The inner core of the plasma $\left(\rho_{N}=0.15\right)$ starts to experience the arrival of the density pulse only $\Delta t \approx 7 m s$ after the injection, reaching its peaked value at a similar time-scale as the electron temperature response $(\Delta t \approx 50 \mathrm{~ms})$, as depicted in Fig. 3c. The change in core electron density is $\Delta n_{e} \approx 6.0 \cdot 10^{17} \mathrm{~m}^{-3}$, which represents only $\sim 4 \%$ of the absolute steady state density. This measurement of electron density in fast time resolution provides evidence to help validate the model discussed in the introduction and in Ref. [29]. A fast increase in the core density, even small in magnitude (and usually difficult to measure), would quickly reduce density gradients and potentially stabilize TEM turbulence, leading to seemingly "nonlocal" transport effects.

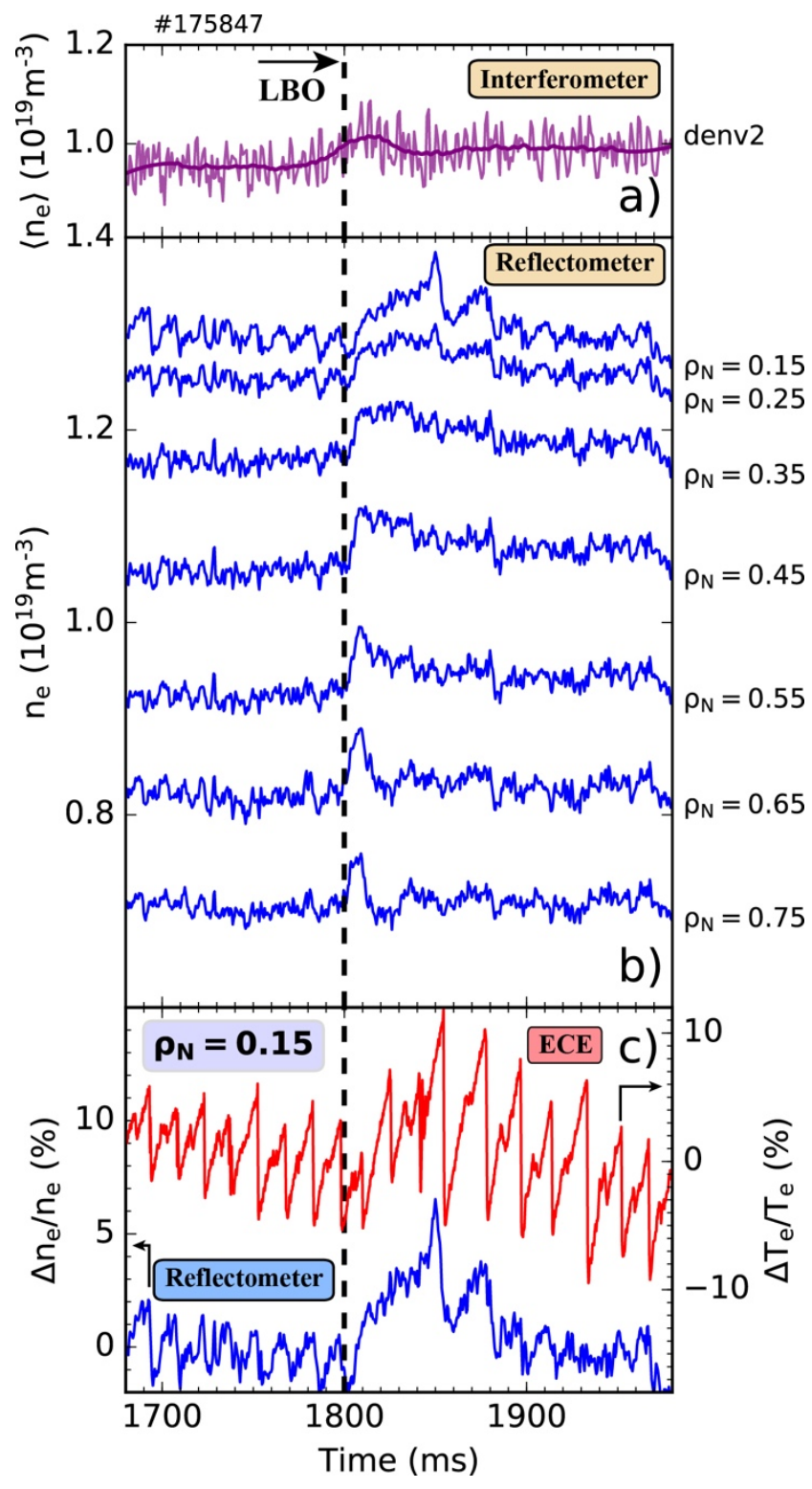

Figure 3. a) Line-average density from vertical $(R=1.94 \mathrm{~m})$ $\mathrm{CO} 2$ interferometer, b) Local density from reflectometer at several radial locations after the injection of impurities at the edge, c) relative change of core electron temperature and density plotted together. 


\section{iii. Behavior of Impurities}

Neutral aluminum particles $\left(\sim 10^{18}\right)$ reach the edge of DIII-D plasmas and get ionized shortly after crossing the last closed flux surface. This local deposition causes a peaked impurity density at the edge ( $\rho_{N} \gtrsim 0.8$ ). The large reversed gradients that build up lead to a strong inward flux of impurities, which reach the core in a very short time, as studied in detail in modeling work at ASDEX Upgrade [37]. The evolution of impurity density following the edge injection can be estimated experimentally using the emission of impurities via soft $x-$ ray diagnostics and the STRAHL impurity transport code [48].

Fig. 4 shows the contribution of the injected impurities to the electron density by quasineutriality (accounting for the evolution of all charge states, $\left.\Delta n_{e}=\sum_{Z} Z \cdot n_{A l, Z}\right)$, compared to the traces of electron density from the reflectometer. Here, impurity density evolution is obtained by running STRAHL simulations within an optimization code to determine maximum likelihood D and $\mathrm{V}$ impurity transport coefficients that match measured absolute levels of soft x-ray emission. The notably good agreement between the two traces in Fig. 4 indicates that the core electron density perturbation can be explained by the arrival of impurities at the core (i.e. deuterium density remains approximately constant). Impurity radiation is

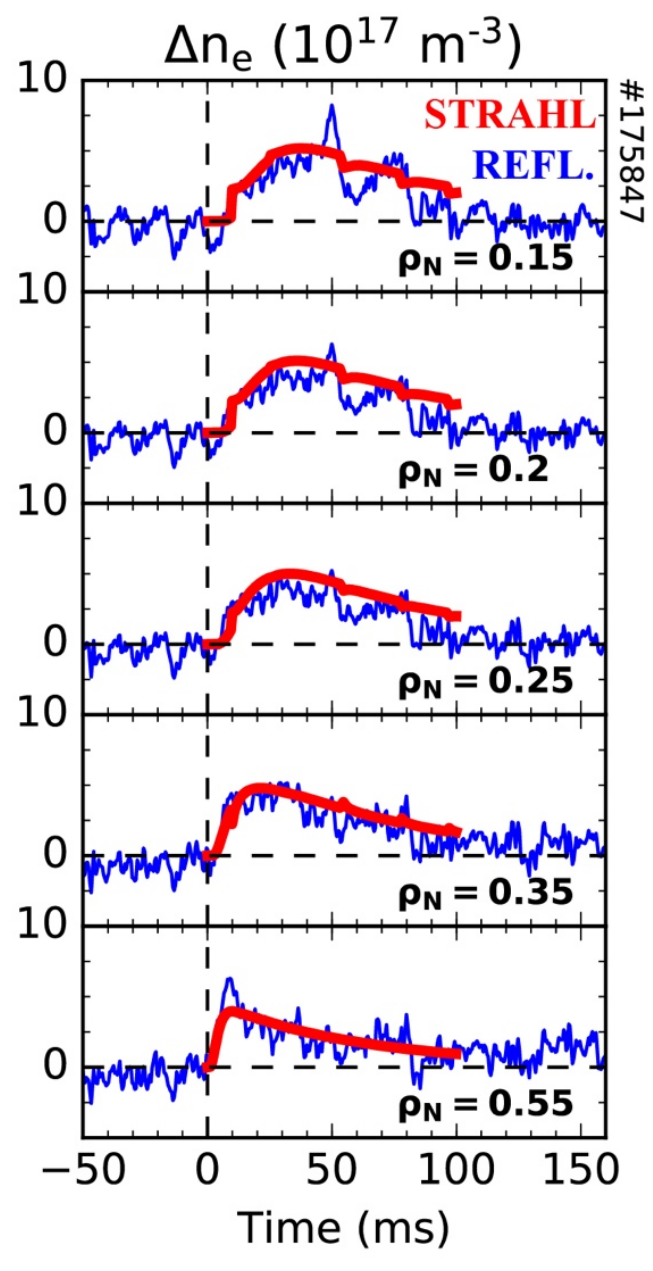

Figure 4. Electron density evolution (blue) from reflectometer and (red) inferred from impurity radiation and STRAHL modeling. only measured for $\rho_{N}<0.6$, and therefore further work is needed to explain changes in electron density in the outer radii. At high density, impurity diffusion is significantly smaller than at low density, further suppressing the stabilization effect of the density gradient reduction.

Cold-pulses (and associated density perturbation) were also triggered by the injection of tungsten in Ohmic DIII-D discharges, but they did not lead to the characteristic temperature inversion at low 
collisionality observed with aluminum. This could be due to much stronger core radiation and ionization power losses for tungsten injections, which dominates core temperature evolution and thus prevents the temperature increase driven by turbulence stabilization. In these experiments, the impurity atoms that reach the plasma become ionized as they travel to the plasma core. For low-Z impurities, most of the radiation from partially ionized states is concentrated at the edge, but high-Z impurities radiate a significant amount of energy in the inner core. Perturbative transport studies then become more complicated because the plasma core is not a source-free region anymore. For this reason, those tungsten injections will not be included in this paper and are left for future work.

\section{Postdictive Modeling of DIII-D Cold-Pulses}

\section{i. Calculation of Steady-State Temperature Profiles}

Steady state electron and ion (Carbon) temperature, and density and toroidal rotation profiles were fitted from experimental measurements with Gaussian Process Regression [49] using the OMFIT framework [50]. CER measurements (via diagnostic neutral beams) were taken after the injection but enough time was allowed, $\Delta t=280 \mathrm{~ms}$, for the profiles to relax back to pre-injection steady-state values. A long time window for the radiation signals $(t=1400-2000 \mathrm{~ms})$ was used to compensate the high noise levels in the bolometer data.

Steady-state predictions were performed using the PT_SOLVER numerical scheme [51] integrated with the TRANSP power balance code [52, 53]. Turbulent transport fluxes were provided by the TGLF-SAT1 [54, 55] quasilinear model with a standard wavenumber grid (normalized to the ion acoustic gyroradius, $\rho_{s}$ ) that accounts for contributions up to $k_{\theta} \rho_{s}=24.0$. Only electron and ion temperatures were evolved, and experimental boundary conditions were set at $\rho_{N}=0.9$ (square

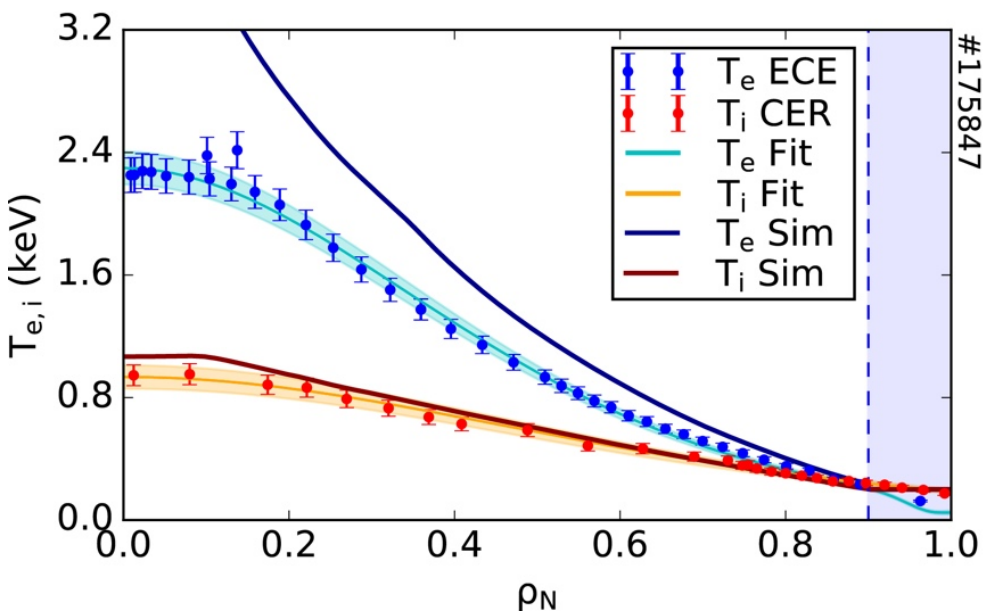

Figure 5. Simulated and experimental electron and ion temperature profiles in steady-state before the cold-pulse injection. Experimental electron temperature is inferred experimentally using an ECE radiometer, and ion temperature is obtained using a CER system via diagnostic neutral beam injections. 
root of the normalized toroidal flux). For simplicity, the rest of simulation settings were identical to those reported in Ref. [29]. Fig. 5 shows that the model over-predicts the steady-state electron temperature. This mismatch did not improve by changes in the boundary conditions nor by enabling density evolution. For completeness, the TGYRO code [56], which uses a different flux-matching solver, was also tested and gave similar predictions. These results in steady-state motivate further work to improve the capabilities of TGLF to predict low-collisionality TEM-dominated regimes.

\section{ii. Perturbative transport calculations}

As done in the past for modeling of Alcator C-Mod plasmas [29], the cold-pulse injection is accompanied by an electron density perturbation. In DIII-D, the fast time resolution reflectometer measurements of electron density can be used to constrain the density pulse that travels from edge to core. Motivated by the results of the impurity transport inferences with STRAHL presented in Section 4iii, deuterium density will be assumed constant during the cold-pulse propagation.

In this study, the reflectometer core density perturbation is fitted to a skewed-Gaussian pulse in space and time. This approach ensures that the density at the plasma core does not increase before edge and middle channels do. This is justified by the fact that the impurities are injected at the edge of the plasma and propagate inwards, thus increasing core density. Interestingly, it is found that the edge and core channels cannot be fitted by a single Gaussian pulse. To resolve this, a correction has been implemented to keep edge density evolution within error bars. The left side of Fig. 6 depicts the raw density data (blue) and the result of two different fits: 1) an edge correction that ensures that the edge density perturbation is within measurement error bars (green, "model A"), and 2) an edge correction that guarantees that density evolution follows the mean of the measurement (red, "model B"). 
The right side of Fig. 6 shows the transient evolution of electron temperature for the two choices of density perturbation. Simulation and experiment are both depicted, evidencing that an experimentally-constrained density perturbation can produce a temperature inversion effect that is close to experimental measurements. Both density pulses give rise to a core temperature increase, but model A provided a larger core temperature increase. This discrepancy between the two is a consequence of a series of events. Around the middle channels $\left(\rho_{N} \sim 0.5-0.3\right)$, the reduction of the density gradient is smaller for model B, which prevents the electron temperature from recovering after the initial drop (as depicted in Fig. 6 at $\rho_{N} \sim 0.35$ ). As a consequence, the inner core of the plasma $\left(\rho_{N} \sim 0.2-0.3\right)$ sees the arrival of higher $a / L_{T e}$, which increases transport and balances the stabilization effect of $a / L_{n}$. Details of the comparison between the two models are presented in the next Section (particularly in Fig. $8)$.
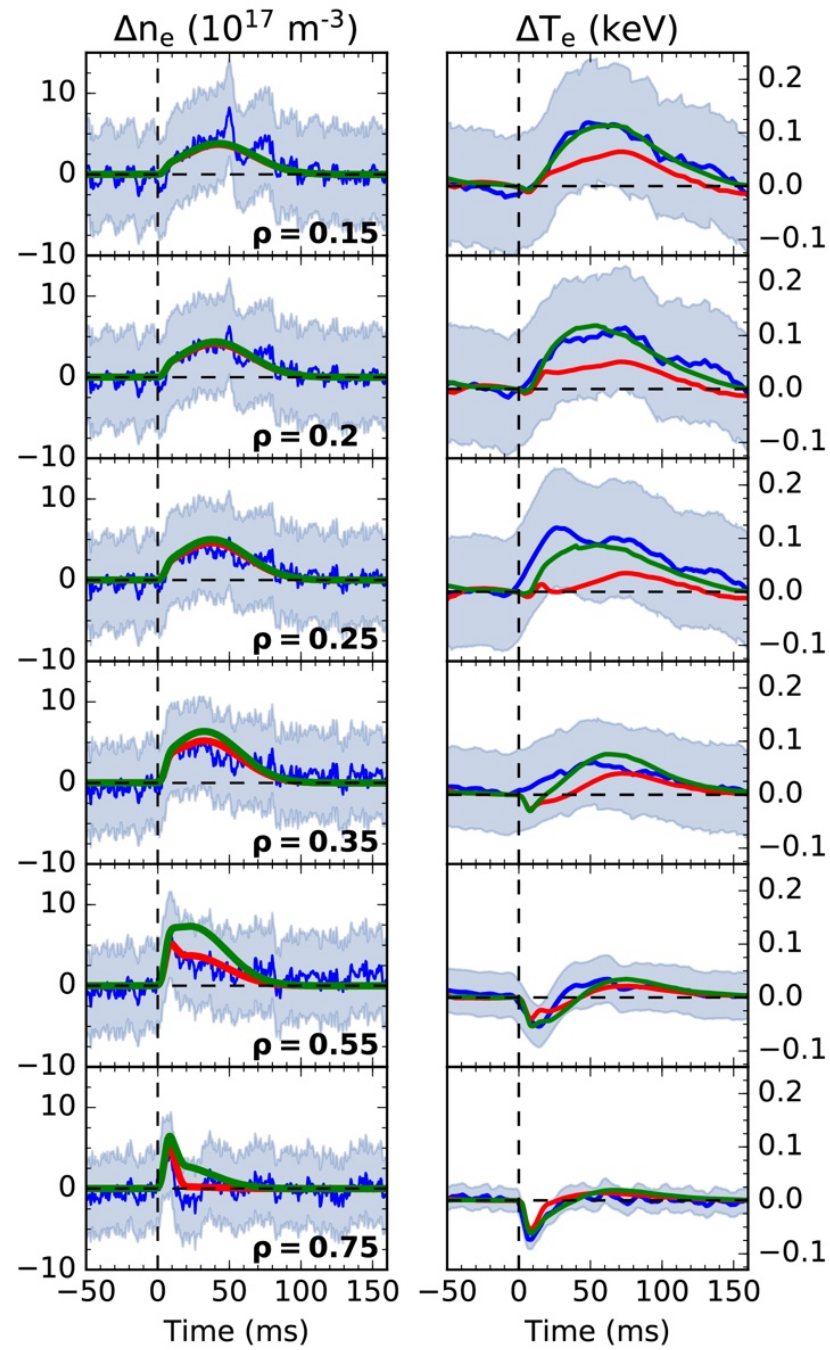

Figure 6. (left) Electron density and (right) temperature for (blue) experiment and (green, red) two different Gaussian-pulse fits. Light blue shaded areas indicate estimated error bars. A better agreement with core temperature evolution is achieved when edge density pulse is larger than the mean of the measurement (yet within error bars). This analysis corresponds to shot \#175847. 


\section{iii. Quasilinear Analysis of Main Turbulence Drives}

During the cold-pulse propagation, TGLF provides electron and ion heat fluxes at all times in order to evolve kinetic profiles self-consistently. Fig. 7 shows the relative importance of each turbulence drive in reducing electron and ion heat fluxes at $\rho_{N}=0.2$ soon after the edge injection $(\Delta t=10 \mathrm{~ms})$. All the qualitative trends are consistent with TEMs as the primary exhaust mechanism for electron heat flux $[57,58]$ : less transport with the reduction of $a / L_{n}$ and $n_{D} / n_{e}$ and more transport with the reduction of $a / L_{T i}$. On the other hand, ITG-driven modes are the main ion heat exhaust mechanism in this plasma (stabilizing effect of $a / L_{T i}$ reduction). Other parameters that have an effect on TEM and ITG mode turbulence, such as $v_{i e}, a / L_{T e}$ and $T_{i} / T_{e}[59,60]$, did not significantly change at this radial location $\left(\rho_{N}=0.2\right)$ at the time plotted $(\Delta t=10 \mathrm{~ms})$.

Most of the TEM stabilization at $\rho_{N}=0.2$ comes from the reduction of density gradients: $\sim 19 \%$ reduction in $a / L_{n e}$ leads to $\sim 65 \%$ lower electron heat flux. During the simulation and these scans, the deuterium density gradient is kept constant and the impurity density gradient changes to fulfill quasineutrality at all times. In order for this to happen, impurities transiently develop a hollowed profile with reversed density gradient $a / L_{n z} \approx-1.0\left(L_{e z} \equiv\right.$ $L_{n e} / L_{n z} \approx-3.0$ ), which acts towards stabilizing TEMs and destabilizing ITG modes. This is consistent with past work on impurity gradient effects on linear gyrokinetic simulations of ITG mode and TEM plasmas [61]. An additional scan was run where $a / L_{n e}=a / L_{n D}=$ $a / L_{n z}$, but it could only account for $\sim 30 \%$ of the total electron heat flux reduction (green dashed line in Fig. 7). Therefore, the strong impurity density gradients that arise as a consequence of the local deposition at the edge and
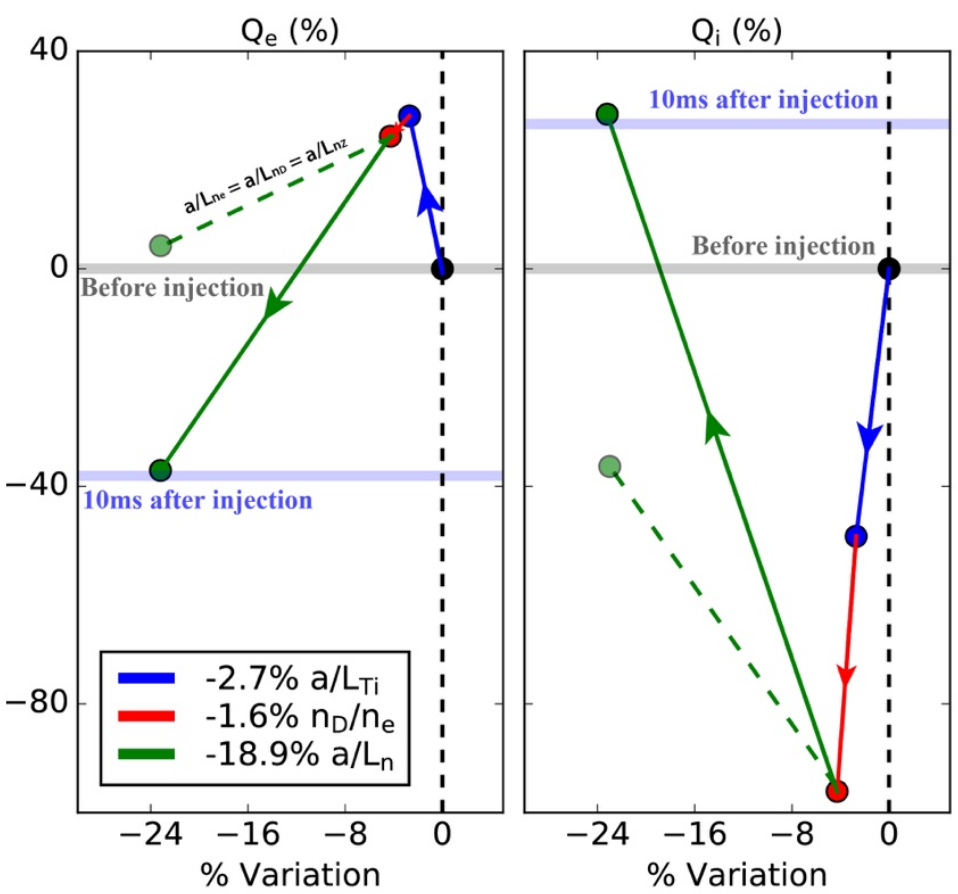

Figure 7. Evolution of the (left) electron and (right) ion heat fluxes (relative to the pre-injection time) as a function of a selection of turbulence drives. Each drive is varied the same amount as in the simulation, 10ms after the injection. Impurity dilution and normalized density gradient are also varied to fulfill quasineutrality during the scans. This analysis corresponds to shot \#175847. 
subsequent inward propagation can be an important stabilization mechanism for TEM turbulence, particularly for plasmas with relatively flat density profiles (as in this case, $\eta_{e} \equiv L_{n e} / L_{T e} \approx 5.0, \varepsilon_{n} \equiv$ $\left.L_{n e} / R \approx 1.0\right)[62]$.

We must highlight that, even though reversed impurity density gradients form after laser blow-off injections, their existence is, in general, not essential for the change in core impurity density gradient to contribute to the temperature inversion. Linear gyrokinetic simulations showed a stabilizing effect of impurity ions on the TEMs regardless of the peaking direction in the small $\eta_{i}=L_{n i} / L_{T i}$ regime [61].

In order to compare model $A$ and model B (results of the temperature evolution in Fig. 6), we explore the behavior of the heat flux with respect to driving gradients at $\rho_{N}=0.35$ (radial position where the differences between models start to become significant). Figs. $8 \mathrm{a}$ and $8 \mathrm{~b}$ show that the primary difference between the two is the stabilization effect of density gradients. For model B, $a / L_{n e}$ is reduced by $\sim 8 \%$ at $\Delta t=30 \mathrm{~ms}$, while for model $\mathrm{A}$ the gradient is reduced by $\sim 18 \%$. Such difference in the gradient is caused by $\sim 4 \%$ absolute density change in model B and $\sim 5 \%$ in model $\mathrm{A}$, highlighting the high sensitivity to small changes in plasma parameters. As shown in Figs. 8c and $8 \mathrm{~d}$, the reduction of $a / L_{n e}$ in model A causes a shift in real frequency of the most unstable linear mode at long wavelength (transition to ITG dominance due to the supression of low-k TEMs), while for model $\mathrm{B}$ there is only a reduction of the TEM linear growth rate, without a dominance transition. Consequently, the reduction of electron heat flux driven at low-k is stronger for model A than model B (Fig. 8e). Figs. 8a and 8b also confirm the stabilizing role of the increase in $T_{i} / T_{e}, Z_{e f f}$ and $v_{i e}$ at $\rho_{N}=0.35$ for both
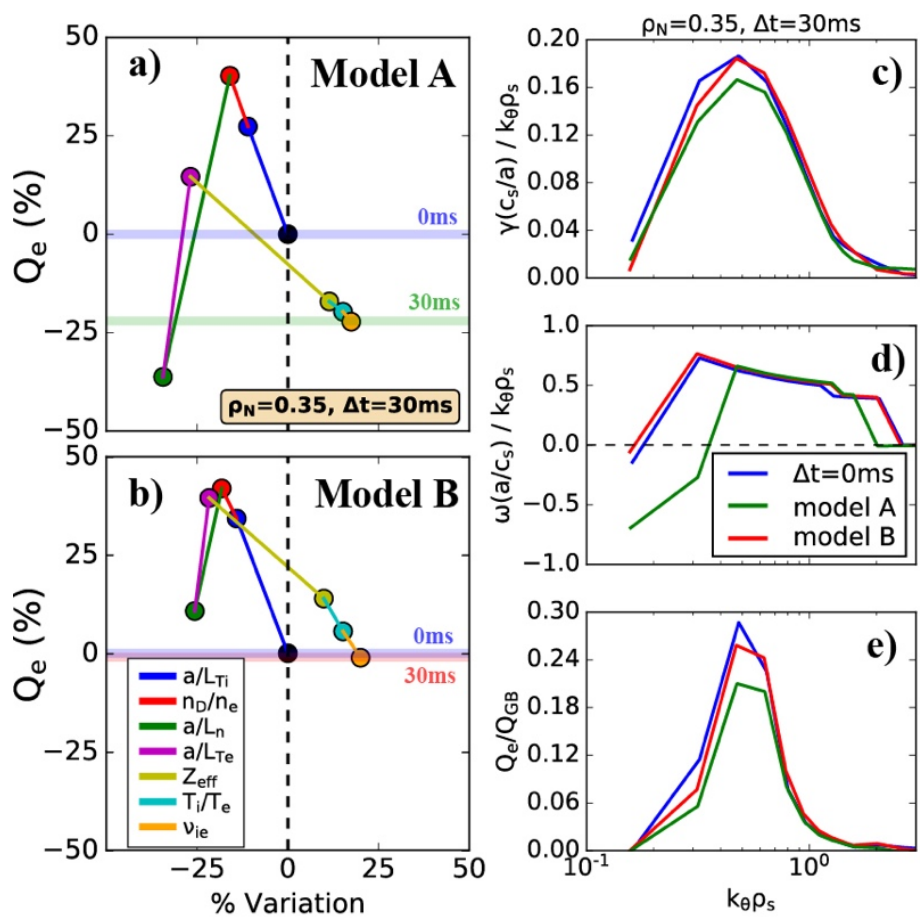

Figure 8. Evolution of the electron heat flux (relative to the pre-injection time) as a function of turbulence drives for a) model $A$ and b) model $B$ at $\rho_{N}=0.35$ and $30 \mathrm{~ms}$ after the injection. Evolution of c) growth rate and d) frequency of the most unstable mode, and e) evolution of the electron heat flux spectrum. This analysis corresponds to shot \#175847. 
models, which helps compensate the destabilization caused by the increased electron temperature gradient at this position.

\section{Conclusions}

In preparation for new DIII-D experiments, empirical predictions and predict-first simulations were both used to identify conditions that would exhibit the two distinct perturbative transport behaviors. The empirical scaling from Refs. [9, 33] was constructed with experimental data from devices other than DIII$\mathrm{D}$, since no evidence of temperature inversions had been reported in this machine. For this reason, theorybased predictions with TGLF were very valuable and helped build confidence that the perturbative transport experiment would run successfully.

The model presented here has been successful in explaining many of the trends observed in previous cold-pulse experiments $[32,37]$, such as the disappearance of the temperature inversion effect at high density, the trend with plasma current, why the temperature flex point seems to move along with rational surfaces, the connection with the thermal coupling between ions and electrons and the effect of auxiliary heating and heat flux ratio. This paper also demonstrates that the model successfully captures the collisionality scaling proposed by [9], and was capable of predicting the cold-pulse behavior in a new machine. High time resolution reflectometer measurements revealed the existence of a density pulse that travels from edge to core, consistent with the inward propagation of impurities after their deposition at the plasma edge. The presence of such a density perturbation was a key element of the original model proposed by [29] to explain the seemingly nonlocal effect observed for many years in magnetically confined plasmas. Not only does this paper provide experimental evidence of rapid impurity and electron density pulses, but it also demonstrates that a big density perturbation is not needed to give rise to the core temperature increase.

Other models have been proposed in past work $[63,64,65,66]$, but their validity to reproduce quantitatively the core temperature behavior and the experimental trends with plasma parameters still remains to be explored. The success of local quasilinear transport models based on nonlinear gyrokinetics (e.g. TGLF and QuaLiKiz) in reproducing multichannel steady-state profiles has been evident for many years $[18,24,25,26,27]$. Our predictive capabilities for future burning plasmas based on these transport models are becoming increasingly accurate thanks to extensive validation studies [67, 68], and this work

provides further confidence that the fundamental assumptions of integrated modeling frameworks based on local physics are enough to reproduce heat transport in tokamak plasmas. 


\section{Acknowledgements}

The authors appreciate insightful discussions with Dr. Mantica, Prof. Gentle and Dr. Citrin on the dynamics of cold-pulse propagation in tokamak plasmas. We thank T.H. Osborne and the DIII-D team for their excellent work on the experiments, and the TRANSP team for their support with the intensive runs. Data analysis was performed using the OMFIT framework [50, 69]. This material is based upon work supported by the U.S. Department of Energy, Office of Science, Office of Fusion Energy Sciences, using the DIII-D National Fusion Facility, a DOE Office of Science user facility, under Awards DE-FC0204ER54698, DE-SC0014264, DE-AC02-09CH11466, DE-FG02-97ER54415, DE-SC0019352, DEFG02-08ER54984. P.R.F. was also supported by Fundación Bancaria "la Caixa" under Award $\mathrm{LCF} / \mathrm{BQ} / \mathrm{AN} 14 / 10340041$. DIII-D data shown in this paper can be obtained in digital format by following the links at https://fusion.gat.com/global/D3D_DMP. Disclaimer: This report was prepared as an account of work sponsored by an agency of the United States Government. Neither the United States Government nor any agency thereof, nor any of their employees, makes any warranty, express or implied, or assumes any legal liability or responsibility for the accuracy, completeness, or usefulness of any information, apparatus, product, or process disclosed, or represents that its use would not infringe privately owned rights. Reference herein to any specific commercial product, process, or service by trade name, trademark, manufacturer, or otherwise does not necessarily constitute or imply its endorsement, recommendation, or favoring by the United States Government or any agency thereof. The views and opinions of authors expressed herein do not necessarily state or reflect those of the United States Government or any agency thereof.

\section{References}

[1] K. W. Gentle, W. L. Rowan, R. V. Bravenec, G. Cima, T. P. Crowley, H. Gasquet, G. A. Hallock, J. Heard, A. Ouroua, P. E. Phillips, D. W. Ross, P. M. Schoch, and C. Watts, Phys. Rev. Lett. 74, 3620 (1995); https://doi.org/10.1103/PhysRevLett.74.3620

[2] M.W. Kissick, J.D. Callen, E.D. Fredrickson, A.C. Janos and G. Taylor, Nucl. Fusion 361691 (1996); https://doi.org/10.1088/0029-5515/36/12/I09

[3] T. Dudok de Wit, M. Erba, M. Mattioli, and J.-L. Ségui, Physics of Plasmas 5, 1360 (1998);

\section{https://doi.org/10.1063/1.872796}


[4] P. Mantica, P. Galli, G. Gorini, G. M. D. Hogeweij, J. de Kloe, N. J. Lopes Cardozo, and RTP Team, Phys. Rev. Lett. 82, 5048 (1999); https://doi.org/10.1103/PhysRevLett.82.5048

[5] F. Rytera, R. Neua, R. Duxa, H.-U. Fahrbacha, F. Leuterera, G. Pereverzeva, J. Schweinzera, J. Stobera, W. Suttropa, ASDEX Upgrade Teama, F. De Lucab, A. Jacchiac and J.E. Kinsey, Nucl. Fusion 401917 (2000); https://doi.org/10.1088/0029-5515/40/11/311

[6] P Mantica, G Gorini, F Imbeaux, J Kinsey, Y Sarazin, R Budny, I Coffey, R Dux, X Garbet, L Garzotti, C Ingesson, M Kissick, V Parail, C Sozzi, A Walden and contributors to the EFDA-JET Workprogramme, Plasma Phys. Control. Fusion 442185 (2002); https://doi.org/10.1088/0741$\underline{3335 / 44 / 10 / 308}$

[7] S. Inagaki, T. Tokuzawa, K. Itoh, K. Ida, S.-I. Itoh, N. Tamura, S. Sakakibara, N. Kasuya, A. Fujisawa, S. Kubo, T. Shimozuma, T. Ido, S. Nishimura, H. Arakawa, T. Kobayashi, K. Tanaka, Y. Nagayama, K. Kawahata, S. Sudo, H. Yamada, and A. Komori (LHD Experiment Group), Phys. Rev. Lett. 107, 115001 (2011); https://doi.org/10.1103/PhysRevLett.107.115001

[8] X.R. Duan, X.T. Ding, J.Q. Dong, Q.W. Yang, L.W. Yan, Yi Liu, X.L. Zou, D.Q. Liu, W.M. Xuan, L.Y. Chen et al, Nucl. Fusion 49104012 (2009); https://doi.org/10.1088/0029-5515/49/10/104012

[9] J.E. Rice, C. Gao, M.L. Reinke, P.H. Diamond, N.T. Howard, H.J. Sun, I. Cziegler, A.E. Hubbard, Y.A. Podpaly, W.L. Rowan et al, Nucl. Fusion 53033004 (2013); https://doi.org/10.1088/0029$\underline{5515 / 53 / 3 / 033004}$

[10] Y.J. Shi, J.M. Kwon, P.H. Diamond, W.H. Ko, M.J. Choi, S.H. Ko, S.H. Hahn, D.H. Na, J.E. Leem, J.A. Lee et al, Nucl. Fusion 57066040 (2017); https://doi.org/10.1088/1741-4326/aa6b23 [11] Yuejiang Shi, Zhongyong Chen, Zhoujun Yang, Peng Shi, Kaijun Zhao, Patrick H. Diamond, JaeMin Kwon, Wei Yan, Hao Zhou, Xiaoming Pan et al, Nucl. Fusion 58044002 (2018); https://doi.org/10.1088/1741-4326/aaaa9b

[12] J D Callen and M W Kissick, Plasma Phys. Control. Fusion 39, B173 (1997); https://doi.org/10.1088/0741-3335/39/12B/014

[13] K. Ida Plasma Phys. Control. Fusion 57044007 (2015); https://doi.org/10.1088/0741$\underline{3335 / 57 / 4 / 044007}$ 
[14] K. Ida, Z. Shi, H.J. Sun, S. Inagaki, K. Kamiya, J.E. Rice, N. Tamura, P.H. Diamond, G. Dif-

Pradalier, X.L. Zou et al, Nucl. Fusion 55013022 (2015); https://doi.org/10.1088/0029-

$\underline{5515 / 55 / 1 / 013022}$

[15] E.J. Doyle (Chair Transport Physics), W.A. Houlberg (Chair Confinement Database and Modelling), Y. Kamada (Chair Pedestal and Edge), V. Mukhovatov (co-Chair Transport Physics), T.H. Osborne (co-Chair Pedestal and Edge), A. Polevoi (co-Chair Confinement Database and Modelling), G. Bateman, J.W. Connor, J.G. Cordey (retired), T. Fujita et al, Nucl. Fusion 47 S18 (2007); https://doi.org/10.1088/0029-5515/47/6/S02

[16] G. M. Staebler, J. E. Kinsey, and R. E. Waltz, Physics of Plasmas 14, 055909 (2007); https://doi.org/10.1063/1.2436852

[17] C. Bourdelle, X. Garbet, F. Imbeaux, A. Casati, N. Dubuit, R. Guirlet, and T. Parisot, Phys. Plasmas 14, 112501 (2007); https://doi.org/10.1063/1.2800869

[18] J.E. Kinsey, G.M. Staebler, J. Candy, R.E. Waltz and R.V. Budny, Nucl. Fusion 51083001 (2011); https://doi.org/10.1088/0029-5515/51/8/083001

[19] R.V. Budny, J.G. Cordey and TFTR Team and JET Contributors, Nucl. Fusion 56, 056002 (2016); https://doi.org/10.1088/0029-5515/56/5/056002

[20] O. Meneghini, P. B. Snyder, S. P. Smith, J. Candy, G. M. Staebler, E. A. Belli, L. L. Lao, J. M. Park, D. L. Green, W. Elwasif, B. A. Grierson, and C. Holland, Physics of Plasmas 23, 042507 (2016); https://doi.org/10.1063/1.4947204

[21] C. Chrystal , B. A. Grierson, G. M. Staebler, C. C. Petty, W. M. Solomon, J. S. deGrassie , K. H. Burrell , T. Tala, and A. Salmi, Physics of Plasmas 24, 056113 (2017);

https://doi.org/10.1063/1.4979194

[22] Jiale Chen, Xiang Jian, Vincent S Chan, Zeyu Li, Zhao Deng, Guoqiang Li, Wenfeng Guo, Nan Shi, Xi Chen and CFETR Physics Team, Plasma Phys. Control. Fusion 59075005 (2017); https://doi.org/10.1088/1361-6587/aa6d20

[23] V.S. Chan, A.E. Costley, B.N. Wan, A.M. Garofalo and J.A. Leuer, Nucl. Fusion 55023017 (2015); https://doi.org/10.1088/0029-5515/55/2/023017

[24] Hyun-Tae Kim, M. Romanelli, X. Yuan, S. Kaye, A.C.C. Sips, L. Frassinetti, J. Buchanan and JET Contributors, 2017 Nucl. Fusion 57066032 (2017); https://doi.org/10.1088/1741-4326/aa6b80 
[25] J Citrin, C Bourdelle, F J Casson, C Angioni, N Bonanomi, Y Camenen, X Garbet, L Garzotti, T Görler, O Gürcan et al, Plasma Phys. Control. Fusion 59124005 (2017); https://doi.org/10.1088/13616587/aa8aeb

[26] O. Meneghini, S.P. Smith, P.B. Snyder, G.M. Staebler, J. Candy, E. Belli, L. Lao, M. Kostuk, T. Luce, T. Luda, J.M. Park and F. Poli, Nucl. Fusion 57086034 (2017); https://doi.org/10.1088/17414326/aa7776

[27] O. Linder, J. Citrin, G.M.D. Hogeweij, C. Angioni, C. Bourdelle, F.J. Casson, E. Fable, A. Ho, F. Koechl, M. Sertoli, the EUROfusion MST1 Team and the ASDEX Upgrade Team, Nucl. Fusion 59 016003 (2019); https://doi.org/10.1088/1741-4326/aae875

[28] N T Howard, C Holland, A E White, M Greenwald, P Rodriguez-Fernandez, J Candy and A J Creely, Plasma Phys. Control. Fusion 60014034 (2018); https://doi.org/10.1088/1361-6587/aa9148 [29] P. Rodriguez-Fernandez, A. E. White, N. T. Howard, B. A. Grierson, G. M. Staebler, J. E. Rice, X. Yuan, N. M. Cao, A. J. Creely, M. J. Greenwald, A. E. Hubbard, J. W. Hughes, J. H. Irby, and F. Sciortino, Phys. Rev. Lett. 120, 075001 (2018); https://doi.org/10.1103/PhysRevLett.120.075001 [30] C. Angioni, A. G. Peeters, F. Ryter, F. Jenko, G. D. Conway, T. Dannert, H. U. Fahrbach, M. Reich, W. Suttrop, and ASDEX Upgrade Team, Physics of Plasmas 12, 040701 (2005); https://doi.org/10.1063/1.1867492

[31] G.D. Conway, C. Angioni, R. Dux, F. Ryter, A.G. Peeters, J. Schirmer, C. Troester, CFN Reflectometry Group and the ASDEX Upgrade team, Nucl. Fusion 46 S799 (2006); https://doi.org/10.1088/0029-5515/46/9/S15

[32] P. Rodriguez-Fernandez, A.E. White, N.T. Howard, B.A. Grierson, X. Yuan, G.M. Staebler, J.E. Rice, C. Angioni, N.M. Cao, A.J. Creely et al (submitted to Nucl. Fusion, IAEA-FEC 2018 special collection)

[33] C. Gao, J.E. Rice, H.J. Sun, M.L. Reinke, N.T. Howard, D. Mikkelson, A.E. Hubbard, M.A. Chilenski, J.R. Walk, J.W. Hughes et al, Nucl. Fusion 54083025 (2014); https://doi.org/10.1088/0029$\underline{5515 / 54 / 8 / 083025}$

[34] J. Luxon Nucl. Fusion 42, 614 (2002); https://doi.org/10.1088/0029-5515/42/5/313 
[35] P. Rodriguez-Fernandez, J.E. Rice, N.M. Cao, A.J. Creely, N.T. Howard, A.E. Hubbard, J.H. Irby and A.E. White, Nucl. Fusion 57074001 (2017); https://doi.org/10.1088/1741-4326/aa6e89

[36] P. Galli, G. Gorini, P. Mantica, G.M.D. Hogeweij, J. de Kloe, N.J. Lopes Cardozo and RTP Team, 1999 Nucl. Fusion 391355 (1999); https://doi.org/10.1088/0029-5515/39/10/301

[37] C. Angioni, E. Fable, F. Ryter, P. Rodriguez-Fernandez, T. Putterich, and the ASDEX Upgrade Team (submitted to Nucl. Fusion)

[38] Hong-Juan Sun, Xuan-Tong Ding, Liang-Hua Yao, Bei-Bin Feng, Ze-Tian Liu, Xu-Ru Duan and Qing-Wei Yang, Plasma Phys. Control. Fusion 52045003 (2010); https://doi.org/10.1088/0741$\underline{3335 / 52 / 4 / 045003}$

[39] X L Zou, A Géraud, P Gomez, M Mattioli, J L Ségui, F Clairet, C De Michelis, P Devynck, T Dudok de Wit, M Erba et al, Plasma Phys. Control. Fusion 421067 (2000); https://doi.org/10.1088/0741-3335/42/10/305

[40] M.W. Kissick, J.D. Callen and E.D. Fredrickson, Nucl. Fusion 38821 (1998) https://doi.org/10.1088/0029-5515/38/6/304

[41] J. E. Rice, M. J. Greenwald, Y. A. Podpaly, M. L. Reinke, P. H. Diamond, J. W. Hughes, N. T. Howard, Y. Ma, I. Cziegler, B. P. Duval et al, Physics of Plasmas 19, 056106 (2012); https://doi.org/10.1063/1.3695213

[42] H. Arnichand, R. Sabot, S. Hacquin, A. Krämer-Flecken, C. Bourdelle, J. Citrin, X. Garbet, J.C. Giacalone, R. Guirlet, J.C. Hillesheim and L. Meneses, Nucl. Fusion 55093021 (2015); https://doi.org/10.1088/0029-5515/55/9/093021

[43] H.J. Sun, P.H. Diamond, Z.B. Shi, C.Y. Chen, L.H. Yao, X.T. Ding, B.B. Feng, X.L. Huang, Y. Zhou, J. Zhou, X.M. Song and the HL-2A team, Nucl. Fusion 51113010 (2011); https://doi.org/10.1088/0029-5515/51/11/113010

[44] M. E. Austin, and J. Lohr, Review of Scientific Instruments 74, 1457 (2003); https://doi.org/10.1063/1.1530387

[45] L. Zeng, G. Wang, E.J. Doyle, T.L. Rhodes, W.A. Peebles and Q. Peng, Nucl. Fusion 46 S677 (2006); https://doi.org/10.1088/0029-5515/46/9/S03 
[46] P. Gohil, K. H. Burrell, R. J. Groebner, and R. P. Seraydarian, Review of Scientific Instruments 61, 2949 (1990); https://doi.org/10.1063/1.1141786

[47] C. Chrystal, K. H. Burrell, B. A. Grierson, S. R. Haskey, R. J. Groebner, D. H. Kaplan, and A. Briesemeister, Review of Scientific Instruments 87, 11E512 (2016); https://doi.org/10.1063/1.4958915

[48] R. Dux 2007 Report IPP 9/82 MPI fur Plasmaphysik, Garching

[49] M.A. Chilenski, M. Greenwald, Y. Marzouk, N.T. Howard, A.E. White, J.E. Rice and J.R. Walk, Nucl. Fusion 55023012 (2015); https://doi.org/10.1088/0029-5515/55/2/023012

[50] O. Meneghini, S.P. Smith, L.L. Lao, O. Izacard, Q. Ren, J.M. Park, J. Candy, Z. Wang, C.J. Luna, V.A. Izzo et al, Nucl. Fusion 55083008 (2015); https://doi.org/10.1088/0029-5515/55/8/083008

[51] X, Yuan, S. Steve, G. Hammett, R. Budny, and G.M. Staebler, APS Division of Plasma Physics (2013); http://adsabs.harvard.edu/abs/2013APS..DPPJP8119Y

[52] J. Breslau, M. Gorelenkova, F. Poli, J. Sachdev and X. Yuan, TRANSP. Computer Sofware (2018); https://doi.org/10.11578/dc.20180627.4

[53] R. Hawryluk, Proceedings of Phys. Plasmas Close to Thermonucl. Cond. (Elsevier, NY, 1979) Vol. 1, p. 19.

[54] G. M. Staebler, J. Candy, N. T. Howard, and C. Holland, Physics of Plasmas 23, 062518 (2016); https://doi.org/10.1063/1.4954905

[55] G.M. Staebler, N.T. Howard, J. Candy and C. Holland, Nucl. Fusion 57066046 (2017); https://doi.org/10.1088/1741-4326/aa6bee

[56] J. Candy, C. Holland, R. E. Waltz, M. R. Fahey, and E. Belli, Phys. Plasmas 16, 060704 (2009); https://doi.org/10.1063/1.3167820

[57] A. G. Peeters, C. Angioni, M. Apostoliceanu, F. Jenko, F. Ryter, and the ASDEX Upgrade team, Physics of Plasmas 12, 022505 (2005); https://doi.org/10.1063/1.1848111

[58] N. Bonanomi, P. Mantica, J. Citrin, C. Giroud, E. Lerche, C. Sozzi, D. Taylor, M. Tsalas, D. Van Eester and JET contributors, Nucl. Fusion 58, 026028 (2018); https://doi.org/10.1088/1741-4326/aa9e7c [59] J. E. Kinsey, R. E. Waltz, and H. E. St. John, Physics of Plasmas 5, 3974 (1998); https://doi.org/10.1063/1.873117 
[60] Alessandro Casati, C. Bourdelle, X. Garbet, and F. Imbeaux, Physics of Plasmas 15, 042310 (2008); https://doi.org/10.1063/1.2906223

[61] Huarong Du, Zheng-Xiong Wang, J. Q. Dong, and S. F. Liu, Physics of Plasmas 21, 052101 (2014); https://doi.org/10.1063/1.4875342

[62] Huarong Du, Zheng-Xiong Wang, and J. Q. Dong, Physics of Plasmas 23, 072106 (2016); https://doi.org/10.1063/1.4955324

[63] V.D. Pustovitov Plasma Phys. Control. Fusion 54124036 (2012); https://doi.org/10.1088/0741$\underline{3335 / 54 / 12 / 124036}$

[64] R O Dendy, S C Chapman and S Inagaki, Plasma Phys. Control. Fusion 55115009 (2013); https://doi.org/10.1088/0741-3335/55/11/115009

[65] F. Hariri, V. Naulin, J. Juul Rasmussen, G. S. Xu, and N. Yan, Physics of Plasmas 23, 052512 (2016); https://doi.org/10.1063/1.4951023

[66] H. Zhu, R. O. Dendy, S. C. Chapman, and S. Inagaki, Physics of Plasmas 22, 062308 (2015); http://dx.doi.org/10.1063/1.4923307

[67] A. J. Creely, N. T. Howard, P. Rodriguez-Fernandez, N. Cao, A. E. Hubbard, J. W. Hughes, J. E. Rice, A. E. White, J. Candy, G. M. Staebler et al, Physics of Plasmas 24, 056104 (2017); https://doi.org/10.1063/1.4977466

[68] P. Rodriguez-Fernandez, A. E. White, A. J. Creely, M. J. Greenwald, N. T. Howard, F. Sciortino and J. C. Wright, Fusion Technol. 74:1-2, 65-76 (2018);

https://doi.org/10.1080/15361055.2017.1396166

[69] B. A. Grierson, X. Yuan, M. Gorelenkova, S. Kaye, N. C. Logan, O. Meneghini, S. R. Haskey, J. Buchanan, M. Fitzgerald, S. P. Smith, L. Cui, R. V. Budny and F. M. Poli, Fusion Technol. 74:1-2, 101115 (2018); https://doi.org/10.1080/15361055.2017.1398585 